\title{
Caracterización de compuestos fenólicos y actividad antioxidante de pulpa de café (Coffea arabica L.) deshidratada de tres fincas cafeteras de la región Amazonas (Perú)
}

\author{
Robert J. Cruzalegui ${ }^{1,2}{ }^{*}$, Orlando Güivin ${ }^{1}$, Armstrong B. Fernández-Jeri ${ }^{1,2}$, y Rosita Cruz $^{2}$ \\ (1) Facultad de Ingeniería y Ciencias Agrarias (FICA), Departamento Académico de Agronomía, Agroindustria y \\ Forestal, Universidad Nacional Toribio Rodríguez de Mendoza de Amazonas, Calle Higos Urco 350, Chachapoyas - \\ Perú. (correo-e: robert.cruzalegui@untrm.edu.pe; armstrong.fernandez@untrm.edu.pe) \\ (2) Instituto de Investigación, Innovación y Desarrollo para el Sector Agrario y Agroindustrial de la Región Amazonas \\ (IIDAA), Calle Higos Urco 350, Chachapoyas - Perú. (correo-e: guivin.agroindustrial.untrm92@gmail.com; \\ rosita.cruz@untrm.edu.pe)
}

* Autor a quien debe ser dirigida la correspondencia

Recibido Mar. 19, 2021; Aceptado May. 17, 2021; Versión final Jul. 12, 2021, Publicado Oct. 2021

\begin{abstract}
Resumen
El objetivo de esta investigación fue caracterizar los compuestos fenólicos y actividad antioxidante de la pulpa de café (Coffea arabica L.) deshidratada a través de dos métodos de secado (aire caliente y liofilizado). Las muestras de pulpa de café se obtuvieron de tres provincias de la región Amazonas (Perú), para luego ser sometidas a un proceso de lavado, oreado, deshidratado, molienda y extracción utilizando agua ultra pura y cuatro solventes orgánicos: etanol $50 \%$, etanol $95 \%$, metanol $50 \%$ y metanol $95 \%$. Se encontró que los extractos obtenidos mediante liofilización y solvente etanol al $95 \%$ tienen la mayor capacidad de extracción, compuestos fenólicos y capacidad antioxidante, con $66.3 \%$ y $402.6 \mathrm{mg}$ equivalentes de ácido gálico/100 g pulpa deshidratada, respectivamente. Se concluye que el etanol al $95 \%$ es el mejor solvente para la extracción de compuestos fenólicos en pulpa de café deshidratada por liofilización.
\end{abstract}

\section{Characterization of phenolic compounds and antioxidant activity of dehydrated coffee pulp (Coffea arabica L.) from three coffee farms in the Amazonas region (Peru)}

\begin{abstract}
The primary objective of this research study was to characterize the phenolic compounds and the antioxidant activity of dehydrated coffee (Coffea arabica L.) pulp by two drying methods (hot air and lyophilization). Coffee pulp samples were obtained from three farms in the Amazonas region (Peru). They were treated in a process that included washing, air drying, dehydration, grinding, and extraction using ultra-pure water and four organic solvents: $50 \%$ ethanol, $95 \%$ ethanol, $50 \%$ methanol, and $95 \%$ methanol. The results showed that the extracts obtained by lyophilization and $95 \%$ ethanol solvent had the most extraction capacity, phenolic compounds, and antioxidant capacity, with $66.3 \%$ and $402.6 \mathrm{mg}$ of Gallic acid equivalents/100 g dehydrated pulp, respectively. It is concluded that $95 \%$ ethanol is the best solvent for the extraction of phenolic compounds in dehydrated coffee pulp by lyophilization.
\end{abstract}




\section{INTRODUCCIÓN}

El café, es una de las bebidas más consumidas en el mundo, contiene varios compuestos bioactivos como los antioxidantes y se considera un alimento funcional (Fonseca-García et al. 2014). Las técnicas de recolección y poscosecha afectan directamente en el valor nutricional y sus componentes bioactivos de diversos alimentos, principalmente del café (Alkaltham et al., 2020). Los compuestos bioactivos se extraen principalmente de fuentes naturales y tienen efectos beneficiosos sobre la salud humana, las frutas y verduras son ricas en diversos componentes como carotenoides, compuestos fenólicos, antioxidantes, vitamina C, entre otros; donde los procesos de extracción de estos compuestos dependen de varios factores como la técnica que se utiliza, la materia prima y el tipo de solvente orgánico empleado (Soquetta et al., 2018).

La producción de cafés especiales para los mercados internacionales involucra características sensoriales particulares de la bebida, así como los sistemas de producción de granos de café, por beneficio húmedo o seco, que determinan los aspectos ambientales como el uso del recurso hídrico y la eliminación de desechos sólidos como la pulpa, mucilago y aguas mieles. Muchos países de todo el mundo tienen problemas con esa eliminación de desechos sólidos pudiéndose producir pigmentos antioxidantes por levaduras con actividades biológicas (Moreira et al., 2018). Diversos estudios han demostrado la presencia de compuestos fenólicos en diferentes residuos producto del beneficio húmedo del café, principalmente en la cáscara y la pulpa del café (Duangjai et al., 2016; Heeger et al., 2018; Gemechu, 2020). También, existen estudios desarrollados en extractos comerciales de frutas de café y muestras en polvo para determinar ácidos clorogénicos (CGA), la cafeína y las actividades antioxidantes (Park, 2013; Kamiyama et al., 2015).

Esquivel y Jiménez (2012) manifestaron que los compuestos fenólicos y los carotenoides son componentes ubicuos de las plantas superiores. Si bien el perfil y las concentraciones de compuestos fenólicos se han estudiado en detalle en las semillas y la infusión de café, el conocimiento sobre su presencia en los subproductos del café (cáscara y pulpa) es más limitado. Esquivel et al., (2020), concluyeron en su trabajo sobre subproductos del café (Coffea arabica L.) como fuente de carotenoides y compuestos fenólicos que el ácido 5- O- cafeoilquínico junto con un hexósido putativo (epi) catequina fueron consistentemente los compuestos fenólicos predominantes entre 16 detectados en la pulpa y cáscara de los granos de café pertenecientes a diferentes variedades con un color de exocarpio distinto. Además, la xantona mangiferina, un agente quimiopreventivo del cáncer, se encontró en todas las muestras, por lo cual recomiendan realizar estudios específicos en la salud en futuras investigaciones.

Díaz et al., (2018) determinaron la retención de metabolitos antioxidantes, su actividad antioxidante y el perfil de taza, de muestras de café pergamino tratadas a diferentes tiempos a una misma temperatura y potencia del tostador, demostrando que el proceso de tostión denominado como grado medio en muestras de café excelso (U.G.Q.), aumentó el contenido de metabolitos secundarios (fenoles totales, flavonoides y taninos condensados) y la actividad antioxidante (ORAC-H, ABTS, DPPH y FRAP), combinado con una calificación de perfil de taza óptima para tiempos de tostión entre 5.5 y $6.5 \mathrm{~min}\left(180^{\circ} \mathrm{C}, 100 \%\right.$ de potencia), otorgándole al café obtenido un equilibrio entre calidad sensorial y propiedades nutracéuticas. El objetivo de esta investigación fue caracterizar la capacidad de extracción de compuestos fenólicos y actividad antioxidante de la pulpa de café variedad catimor deshidratada, proveniente de tres fincas cafetaleras mediante la utilización de agua ultra pura y los solventes orgánicos etanol $50 \%$, etanol $95 \%$, metanol $50 \%$ y metanol $95 \%$.

\section{METODOLOGÍA}

Los materiales y métodos se presentan a continuación en nueve subsecciones donde se describe desde la recolección de las muestras ubicadas en tres provincias de la región Amazonas-Perú hasta el análisis de datos.

\section{Recolección de muestras}

Se recolectó muestras de $6 \mathrm{~kg}$ de café (Coffea arabica L. var. Catimor), provenientes de tres fincas cafetaleras ubicadas en las provincias de Rodríguez de Mendoza, distrito San Nicolás, Sector Onche (1673 msnm), Utcubamba, distrito Bagua Grande, Sector San Juan (1690 msnm) y Luya, distrito Camporredondo, Sector Ocsho (1719 msnm), que se encuentran en la región Amazonas. Las muestras recolectadas estuvieron en estado de madurez comercial, descartando frutos sobre maduros y verdes, para ser finalmente colocados los granos de café en recipientes enfriadores cerrado herméticamente (con capacidad de 8 - $11 \mathrm{~L}$ ), conteniendo paquete de gel congelado, paño absorbente y bolsa polietileno, de esta manera manteniendo las condiciones adecuadas de conservación de los frutos de café durante su traslado al laboratorio de Ingeniería de Alimentos de la Universidad Nacional Toribio Rodríguez de Mendoza de Amazonas (UNTRM).

\section{Acondicionamiento de la pulpa de café}

Los frutos de café fueron sometidos a molienda en un molino semi abierto marca Imsa-Perú con el objetivo de separar la pulpa del pergamino y luego realizar el retiro del mucílago adherido a la pulpa mediante un lavado con 
agua potable a alta presión. La pulpa lavada se oreó durante una hora a temperatura ambiente para remover el agua parcialmente y luego se procedió a la deshidratación por aire caliente y liofilizado (Peña-Aguilar et al., 2017).

\section{Secado por aire caliente}

La pulpa de café se secó en una estufa de secado con circulación de aire natural marca Ecocell por 48 a $50{ }^{\circ} \mathrm{C}$, para obtener una humedad final entre 8-10\% y peso constate. La pulpa deshidratada se trituró en un molino triturador Fischer Agro-Perú y luego se tamizó utilizando un tamiz UVR-USA con huecos no mayor a 2 mm. La humedad de pulpa café se determinó por gravimetría con tal fin en la pulpa de café en una balanza analítica Nimbus fue pesado antes y después de su desecado en un desecador de vidrio 250MM Marca MullerAlemania, luego el porcentaje de la humedad se calculó por diferencia de pesos medido en un analizador de humedad Mettler Toledo HX204 (Tran et al., 2020).

\section{Liofilización}

La pulpa se deshidrató utilizando el procedimiento fue desarrollado por Reichembach y De Oliveira (2020) con algunas modificaciones, donde las muestras de pulpa fueron congeladas entre -150 y $-180{ }^{\circ} \mathrm{C}$ con nitrógeno líquido, para luego ser liofilizadas en un Liofilizador Labconco modelo 72040-USA bajo las condiciones de operación: temperatura del colector $-85.8^{\circ} \mathrm{C}$, temperatura inicial del congelador $-2.58{ }^{\circ} \mathrm{C}$, y vacío a presión de 0.102 mbar por un tiempo de $48 \mathrm{~h}$. La pulpa de café liofilizado se trituró un molino triturador Fischer Agro-Perú y tamizó en un tamiz con huecos no mayor a $2 \mathrm{~mm}$ después del secado.

\section{Determinación de la capacidad de extracción de compuestos antioxidantes}

Se empleó el método de extracción líquido-sólido convencional modificado para pulpa de café (Chirinos et al., 2007; Aboshora et al., 2014). Para la extracción se utilizó agua ultra pura y solventes orgánicos: etanol 50 \%, etanol $95 \%$, metanol $50 \%$ y metanol $95 \%$, con una relación de 1:20 w/v (g de muestra/ml de solvente), donde se procedió a pesar $0.5 \mathrm{~g}$ de muestra para mezclar con $10 \mathrm{ml}$ de cada solvente correspondiente. Estas mezclas se realizaron en dos proporciones, con la ayuda de un agitador y mezclador orbital se agitó a 200 rpm en la oscuridad durante $3 \mathrm{~min}$ a temperatura ambiente de $20^{\circ} \mathrm{C}$. Luego las mezclas se centrifugaron a $5000 \mathrm{rpm}$ durante $10 \mathrm{~min}$ y se recogieron los sobrenadantes con papel filtro de paso lento. Los residuos de pulpa de café se volvieron a extraer adicionándole solvente una cantidad de $5 \mathrm{ml}$, se agitó con el agitador y mezclador por 5 min y luego se llevó a centrifugar. Se recogieron los sobrenadantes separados lentamente con papel filtro para luego nuevamente centrifugar y el sobrenadante obtenido se analizó. En las repeticiones adicionales se trabajó bajo las mismas condiciones. Los sobrenadantes de cada extracción se almacenaron a $-20^{\circ} \mathrm{C}$ para su análisis en compuestos fenólicos totales (TP) y actividad antioxidante (AA).

\section{Determinación de fenoles totales}

El contenido de fenoles totales se determinó usando el método de Folin - Ciocalteu con algunas modificaciones (Singleton et al., 1999; Kim et al., 2016). Los extractos de pulpa de café (50 uL) se mezclaron con 2500 uL de reactivo de Folin-Ciocalteu, $450 \mathrm{uL}$ de agua pura y 2000uL de solución de carbonato sódico (7.5 \% p = v). La mezcla se agitó en un agitador y mezclador orbital por $10 \mathrm{~s}$. La mezcla almacenó a temperatura ambiente durante $30 \mathrm{~min}$ a oscuras. La absorbancia se midió a $760 \mathrm{~nm}$ frente a un blanco. Se usaron soluciones acuosas de ácido gálico para la calibración, los resultados se expresan como mg equivalentes de ácido gálico.

\section{Determinación de actividad antioxidante}

La actividad antioxidante de los extractos de pulpa de café se determinó mediante la medida de la absorbancia de los radicales 2,2-difenil-1-picrilhidrazilo - DPPH (Kuskoski et al., 2005; Kim et al., 2016). Para ello se preparó $100 \mathrm{ml}$ de solución DPPH (2,2-difenil-1-picril hidrazilo) en metanol de $50 \mathrm{mg} / \mathrm{L}$. Luego se preparó una solución de metanol y los extractos de pulpa de café en una concentración de $300 \mu \mathrm{g} / \mathrm{ml}(600 \mu \mathrm{L}$ de muestra/2 ml metanol), denominada solución A. Además, el blanco de muestra se preparó con $0.75 \mathrm{ml}$ de muestra de la solución A y 1.5 $\mathrm{ml}$ de metanol. Se preparó el patrón de referencia con $1.5 \mathrm{ml}$ de DPPH y $0.75 \mathrm{ml}$ de agua ultra pura. Posteriormente se preparó la muestra con $0.75 \mathrm{ml}$ de la solución A y $1.5 \mathrm{ml}$ de DPPH, con concentración final de $100 \mu \mathrm{g} / \mathrm{ml}$, dejándose por $5 \mathrm{~min}$ en oscuridad. Finalmente se tomó lectura a $517 \mathrm{~nm}$ en un espectrofotómetro UVline 9400 Secoman-Francia. Con los datos de la absorbancia se calculó el porcentaje de captación de radicales libres (Kuskoski et al., 2005; Díaz et al., 2018). Para ello se utilizó la siguiente ecuación:

Actividad Antioxidante $=\left[1-\frac{\mathrm{A} 2-\mathrm{A} 3}{\mathrm{~A} 1}\right] * 100$

Donde, $A_{1}$ : Absorbancia del patrón de referencia; $A_{2}$ : Absorbancia de la muestra; $A_{3}$ : Absorbancia del blanco de muestra. 


\section{Análisis de datos}

Para evaluar las diferencias estadísticas significativas entre las medias de la capacidad de extracción, fenoles totales y actividad antioxidante en muestras obtenidas de tres fincas cafetaleras ubicadas en tres provincias de la región Amazonas, se empleó el análisis de varianza (ANDEVA) y comparaciones múltiples de Tukey, con un nivel de significancia de 0.05 utilizando el paquete estadístico SPSS Statistics 25.

\section{RESULTADOS Y DISCUSIÓN}

Los resultados se presentan en tres secciones i) Determinación de la capacidad de extracción, ii) Determinación de fenoles totales y iii) Determinación de la actividad antioxidante.

\section{Determinación de la capacidad de extracción (CE)}

La capacidad de extracción se presenta en la Tabla 1, el extracto de pulpa de café de la provincia Rodríguez de Mendoza tuvo mayor CE respecto a las muestras de las demás provincias.

Tabla 1: Valores promedio de capacidad de extracción de pulpa de café

\begin{tabular}{|c|c|c|c|}
\hline Lugar de procedencia & Tipo de deshidratado & Tipo de solvente & Capacidad de extracción (\%) \\
\hline \multirow[t]{10}{*}{ Rodríguez de Mendoza } & \multirow[t]{5}{*}{ Aire Caliente } & Agua UP & $53.0 \pm 0.57$ \\
\hline & & Etanol $50 \%$ & $62.3 \pm 0.91$ \\
\hline & & Etanol $95 \%$ & $71.7 \pm 0.74$ \\
\hline & & Metanol $50 \%$ & $68.3 \pm 0.71$ \\
\hline & & Metanol $95 \%$ & $67.0 \pm 0.00$ \\
\hline & \multirow[t]{5}{*}{ Liofilización } & Agua UP & $57.3 \pm 0.67$ \\
\hline & & Etanol $50 \%$ & $55.3 \pm 0.88$ \\
\hline & & Etanol $95 \%$ & $67.3 \pm 0.35$ \\
\hline & & Metanol $50 \%$ & $66.7 \pm 0.35$ \\
\hline & & Metanol $95 \%$ & $69.3 \pm 0.95$ \\
\hline \multirow[t]{10}{*}{ Utcubamba } & \multirow[t]{5}{*}{ Aire Caliente } & Agua UP & $59.0 \pm 0.58$ \\
\hline & & Etanol $50 \%$ & $62.0 \pm 0.00$ \\
\hline & & Etanol $95 \%$ & $67.3 \pm 0.70$ \\
\hline & & Metanol $50 \%$ & $67.3 \pm 0.35$ \\
\hline & & Metanol $95 \%$ & $70.3 \pm 0.96$ \\
\hline & \multirow[t]{5}{*}{ Liofilización } & Agua UP & $55.0 \pm 0.58$ \\
\hline & & Etanol $50 \%$ & $57.3 \pm 0.33$ \\
\hline & & Etanol $95 \%$ & $62.3 \pm 0.90$ \\
\hline & & Metanol $50 \%$ & $59.3 \pm 1.35$ \\
\hline & & Metanol $95 \%$ & $60.0 \pm 0.00$ \\
\hline \multirow[t]{10}{*}{ Luya } & \multirow[t]{5}{*}{ Aire Caliente } & Agua UP & $55.3 \pm 0.88$ \\
\hline & & Etanol $50 \%$ & $65.7 \pm 0.70$ \\
\hline & & Etanol $95 \%$ & $74.0 \pm 0.65$ \\
\hline & & Metanol $50 \%$ & $68.7 \pm 0.36$ \\
\hline & & Metanol $95 \%$ & $67.7 \pm 0.35$ \\
\hline & \multirow[t]{5}{*}{ Liofilización } & Agua UP & $49.7 \pm 0.33$ \\
\hline & & Etanol 50 \% & $52.3 \pm 0.33$ \\
\hline & & Etanol $95 \%$ & $59.3 \pm 0.67$ \\
\hline & & Metanol $50 \%$ & $54.0 \pm 0.57$ \\
\hline & & Metanol $95 \%$ & $47.3 \pm 0.33$ \\
\hline
\end{tabular}

La CE presenta diferencias estadísticamente significativas $(p<0.05)$ de sus medias en grupos de lugar de procedencia y tipo de deshidratado de pulpa de café con todos sus tratamientos. La pulpa de café sometida por aire caliente tuvo mayor $\mathrm{CE}$ con respecto al proceso de liofilización mientras que la pulpa de café extraídos con solvente etanol $95 \%$ tuvo mayor CE con respecto a los demás solventes y fue mayor cuando se aplicó la deshidratación en secado con aire caliente. Los resultados de capacidad extracción estuvieron relacionados con la procedencia de la muestra, tipo de deshidratado y tipo de solvente, la CE fue mayor para las muestras procedentes de la provincia de Luya en estufa $(74.0 \pm 0.65 \%)$ seguido de Rodríguez de Mendoza en liofilizado 
(69.3 $\pm 0.95 \%)$, esto se debería a la altitud del cultivo de café donde fue recolectada siendo menor altitud la provincia Rodríguez de Mendoza (1673 msnm), menor respecto a Luya. Así mismo, la deshidratación por liofilización fue mayor cuando se empleó metanol $95 \%$; es posible que la polaridad del solvente orgánico estuvo relacionada con la mayor remoción del agua; así mismo los contenidos fenólicos, antioxidantes y propiedades nutritivas se encuentran en la pulpa de café liofilizada. Al respecto, durante este proceso de lixiviación fue determinante la polaridad y concentración del solvente orgánico; las características químicas de los antioxidantes y su mayor solubilidad se ha dado con metanol y etanol que son menos polares que el agua (Gouri et al. 2017); también, la liofilización no afecta la estabilidad y la actividad de compuestos bioactivos, se logra la disminución de la actividad de agua, mejoramiento de la textura y aumento de la porosidad producida por la sublimación de los cristales de hielo (Sema-Cock et al. 2015). El solvente con mayor capacidad extracción fue cuando se empleó etanol $95 \%$ en muestras procedentes de Utcubamba y Rodríguez de Mendoza seguido de metanol 95 \% para Rodríguez de Mendoza, los resultados obtenidos son cercanos con los reportados en investigaciones desarrolladas (Esquivel et al., 2020; Aboshora et al., 2014) en subproductos del café (Coffea arabica L.) y en la fruta Hyphaene Thebaica L. Mart (Arecaceae) (Doum), respectivamente.

\section{Determinación de Fenoles Totales (FT)}

Las Figuras 1, 2 y 3 representan los FT expresados en mg equivalente ácido gálico/100g pulpa deshidratada. El extracto de pulpa café proveniente de, la provincia Utcubamba tuvo mayor contenido de FT con respecto a las demás provincias. La pulpa de café liofilizada tuvo mayor contenido de FT con respecto al secado por estufa. EI solvente etanol $95 \%$ fue mejor en el proceso de extracción del contenido FT, respecto a los demás solventes, siendo mayor para las muestras procedentes de Utcubamba y liofilizadas. El contenido de FT presenta diferencias estadísticamente significativas $(p<0.05)$ de sus medias entre los tipos de deshidratado en grupos para lugar de procedencia y tipo de deshidratado de pulpa de café.

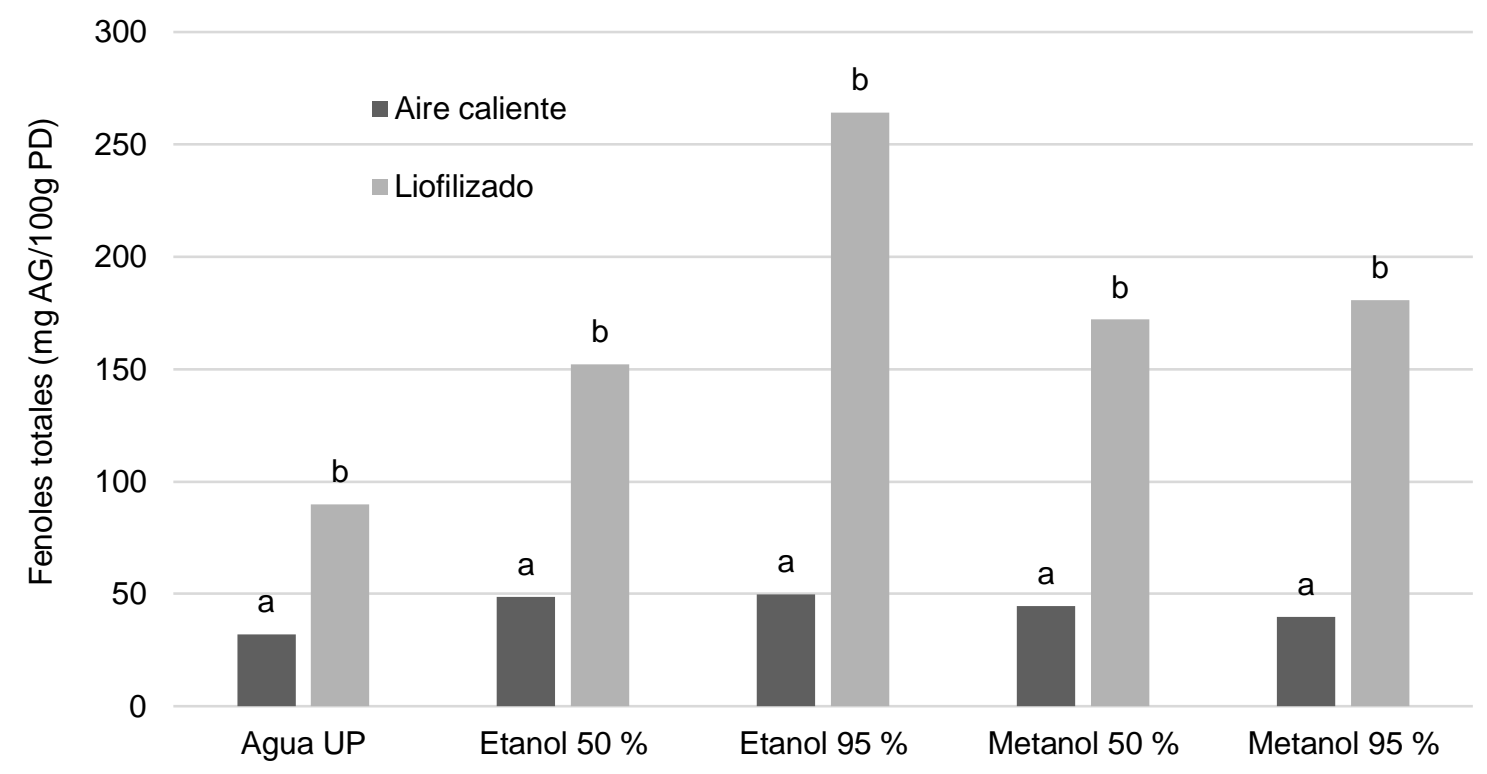

Tipos de solventes según concentraciones

Fig. 1: Fenoles totales de pulpa de café procedente de Rodríguez de Mendoza. Dentro de cada solvente, barras con letras diferentes indican diferencias significativas

Comparando los resultados del contenido fenólico de las muestras de diferentes procedencias se encontró un mayor contenido de fenoles totales en la liofilización, la provincia Utcubamba tuvo $479.5 \mathrm{mg} \mathrm{AG/100g} \mathrm{PD} \mathrm{(Figura}$ 2) seguido de la provincia de Rodríguez de Mendoza con $264.3 \mathrm{mg} \mathrm{AG/100} \mathrm{g} \mathrm{PD} \mathrm{(Figura} \mathrm{1).} \mathrm{Serna} \mathrm{et} \mathrm{al.} \mathrm{(2018),}$ consideran que la pulpa de café es una materia seca de alto contenido de polifenoles, esto es debido al manejo agronómico del cultivo, tipo de suelo, altitud, clima. La pulpa de café liofilizada presentó mejor contenido de fenoles totales cuando se empleó metanol $95 \%$ respecto a la obtenida por aire caliente, esto puede ser a la pulpa de café fue sometida a un secado por estufa a temperatura caliente y tiempo de proceso en presencia de oxígeno ambiental provocando la disminución de la concentración de compuestos fenólicos.

Para el caso de la muestra proveniente de Luya (Figura 3) como en el caso de las otras provincias donde se recolectaron café, es con el proceso de deshidratación por liofilización la mayor obtención de los FT (230.4 mg AG/100 g PD). La cantidad de FT tiene una relación con la mayor CE y en general es con etanol $95 \%$ respecto a los demás solventes que se extrajo más FT, estos resultados son superiores a los encontrados por Vega et al., 
(2017) en la determinación del contenido de polifenoles totales, flavonoides y actividad antioxidante de 34 cafés comerciales de Panamá. Estos valores están dentro del rango de $37-55 \mathrm{mg} / \mathrm{g}$. esto puede ser debido a la polaridad del solvente utilizado para la extracción en este estudio. También, Shushant et al., (2019) mencionan que los componentes antioxidantes como moléculas de fenoles, carotenos y flavonoides se encargan de desactivar los radicales libres en función de su capacidad para donar átomos de hidrógeno a los radicales libres. Hay una correlación entre el contenido total de fenólicos y flavonoides con la capacidad antioxidante en extractos de plantas.

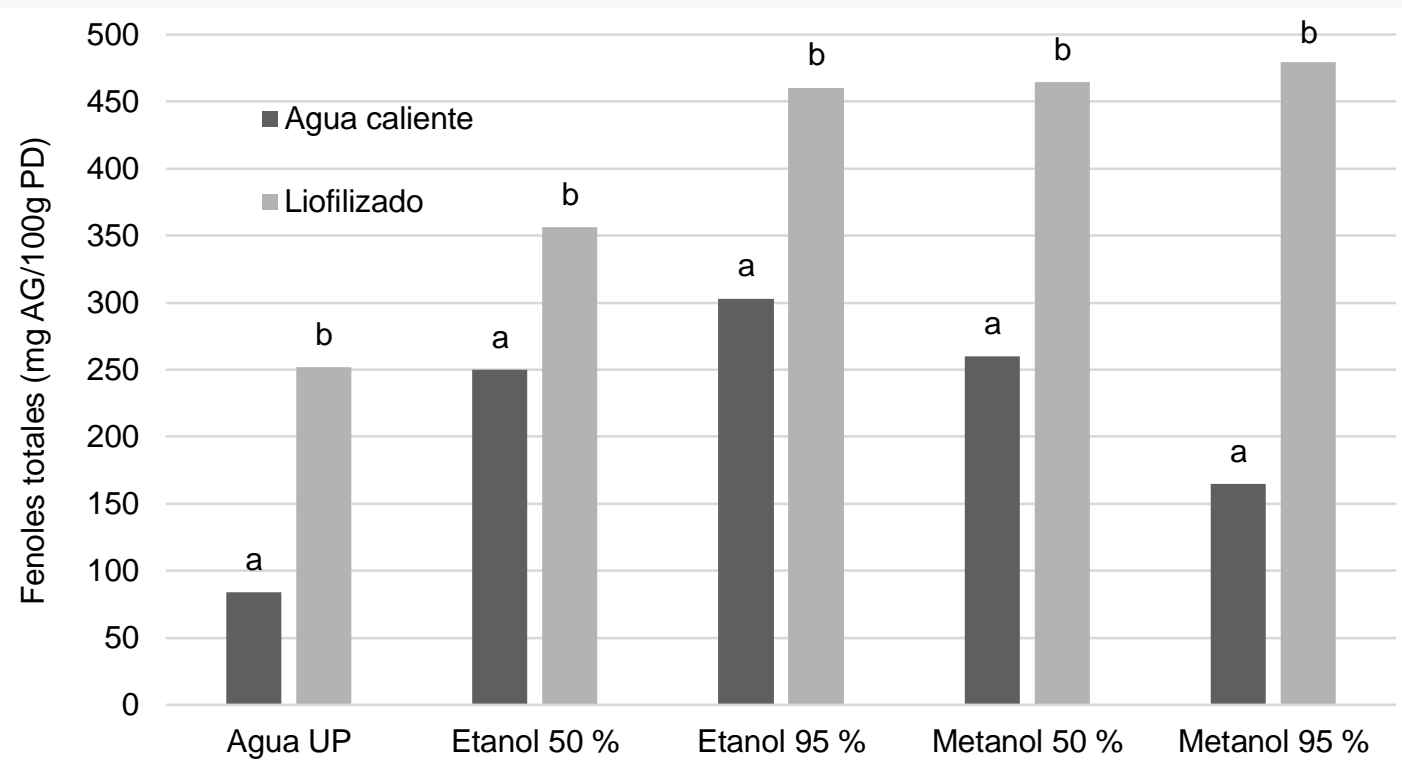

Tipos de solventes según concentraciones

Fig. 2: Fenoles totales de pulpa de café procedente de Utcubamba. Para cada solvente, barras con letras diferentes indican diferencias significativasv

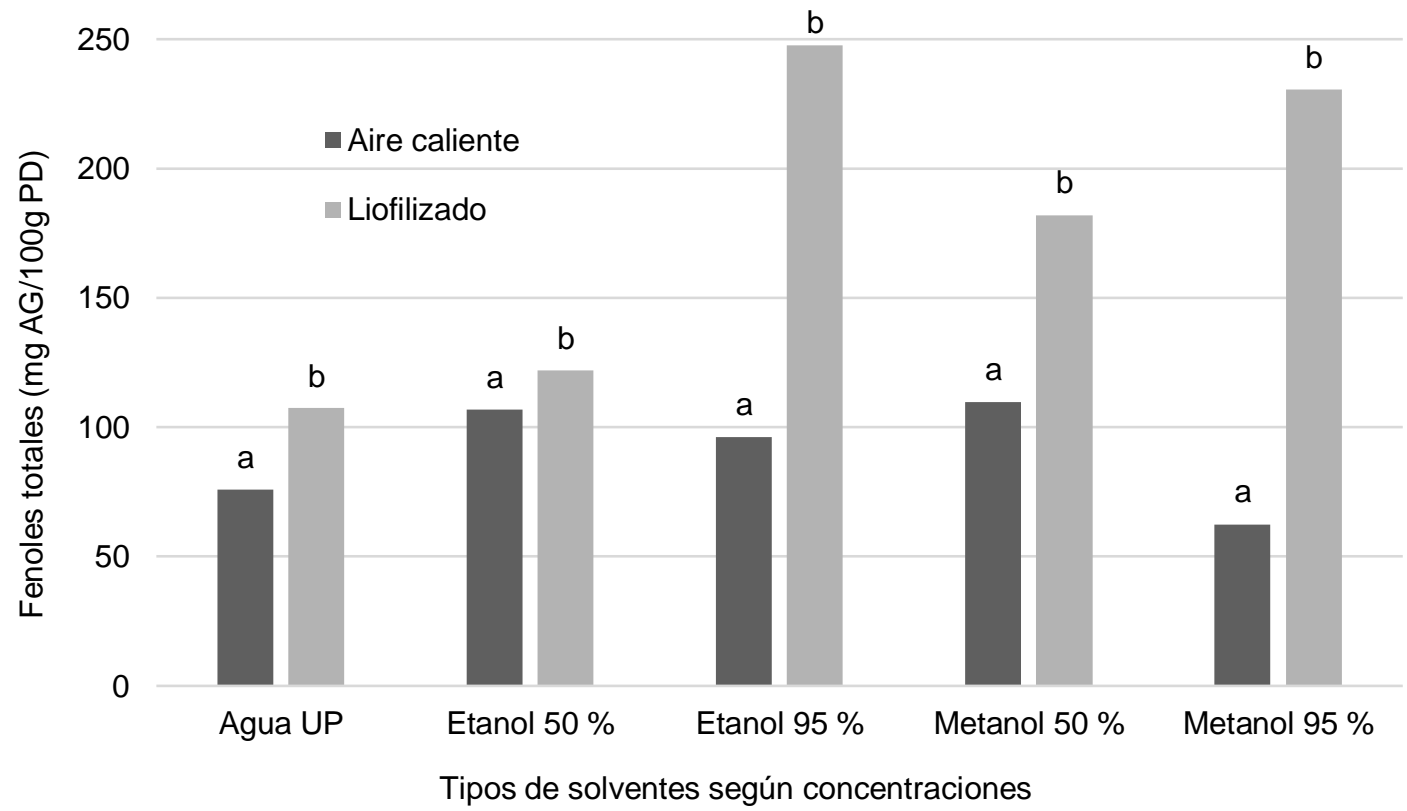

Fig. 3: Fenoles totales de pulpa de café procedente de Luya. Para cada solvente, barras con letras diferentes indican diferencias significativas

\section{Determinación de Actividad Antioxidante (AA)}

De acuerdo a las Figuras 4, 5 y 6 el extracto de pulpa de café proveniente de la provincia Utcubamba tiene alta AA con respecto a las demás. Se observó que no existe diferencia significativa en el tipo deshidratado. La pulpa café alcanzó mayor AA extraídos con metanol $95 \%$, con respecto a los demás solventes. 


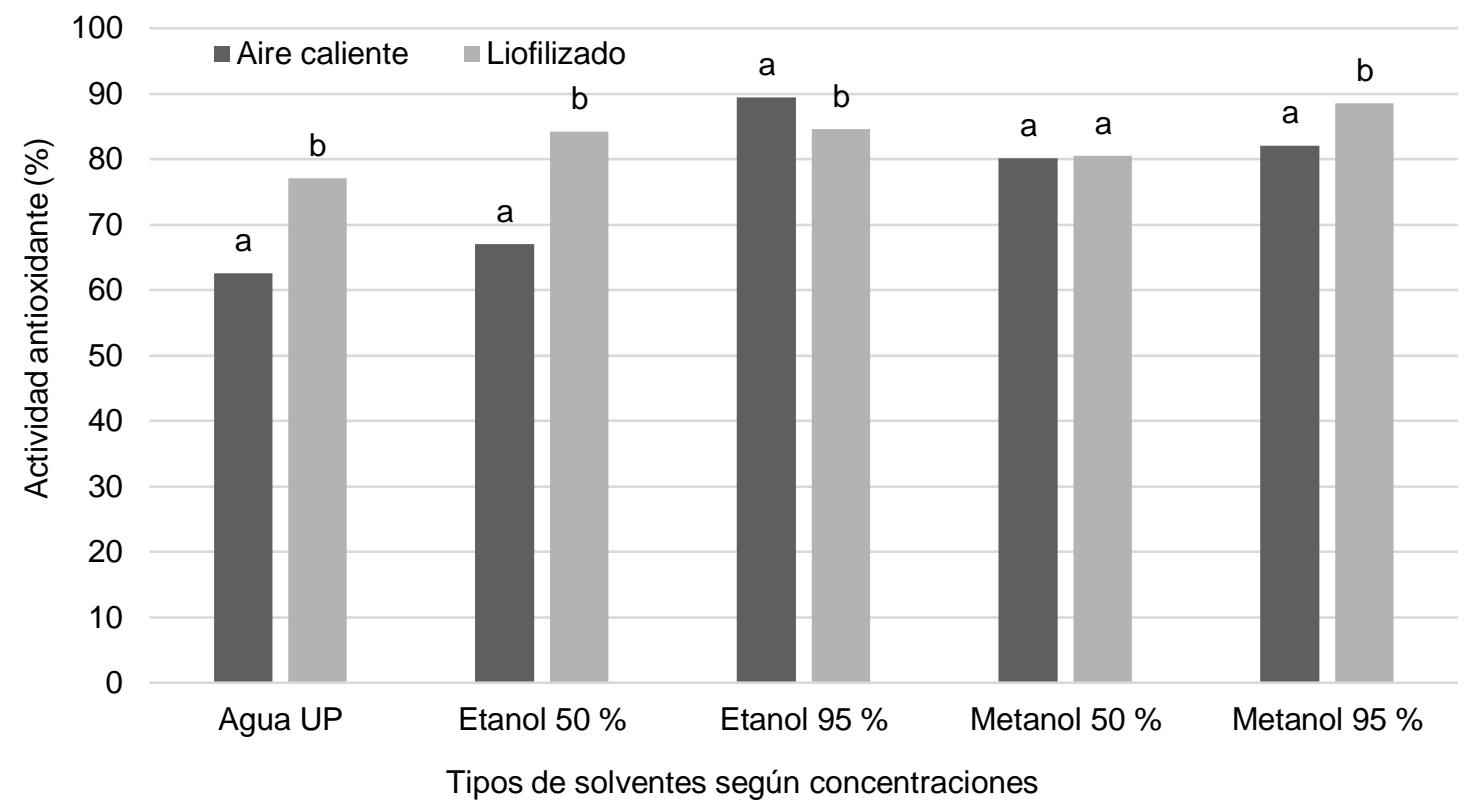

Fig. 4: Actividad antioxidante de pulpa de café procedente de Rodríguez de Mendoza. Para cada solvente, barras con letras diferentes indican diferencias significativas

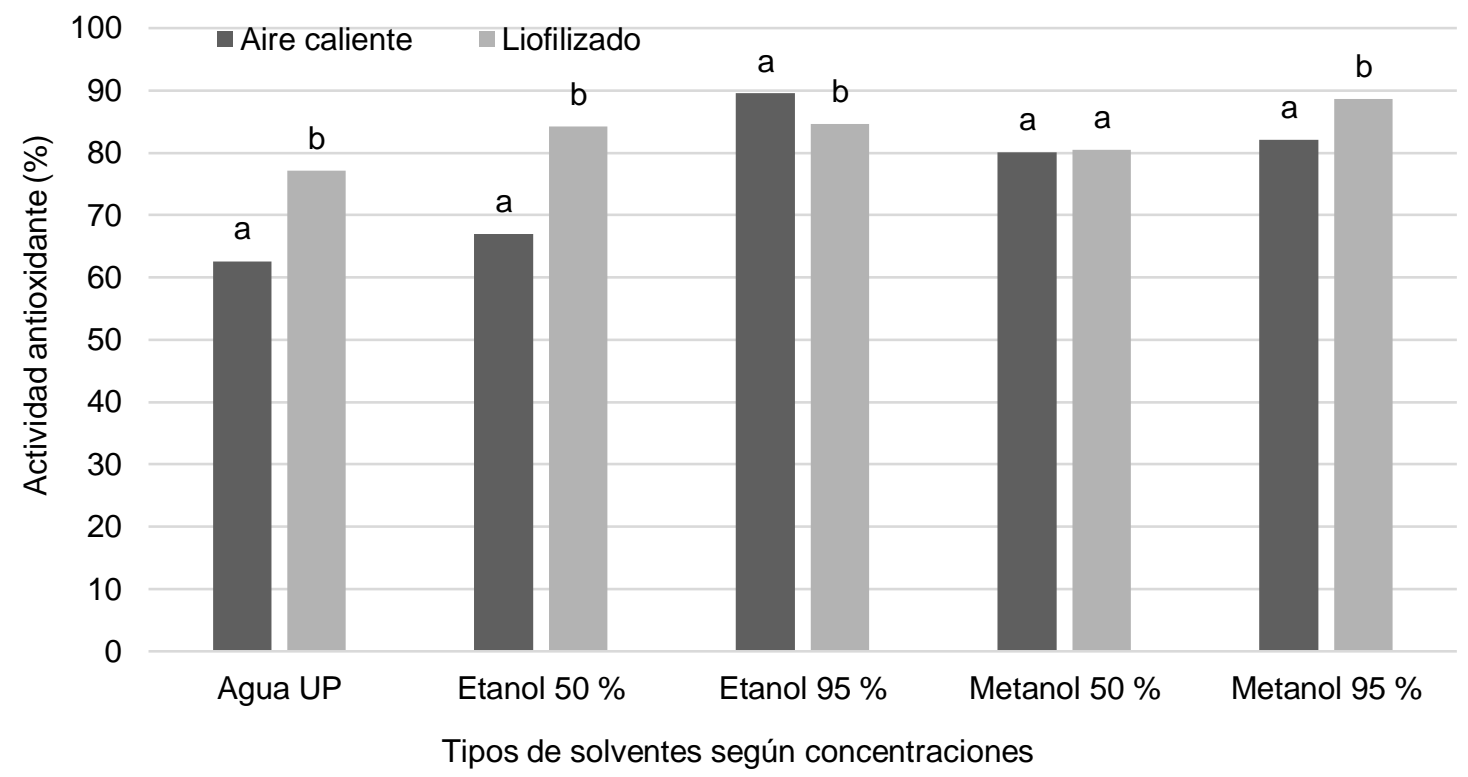

Fig. 5: Actividad antioxidante de pulpa de café procedente de Utcubamba. Para cada solvente, barras con letras diferentes indican diferencias significativas

El contenido de AA presenta diferencias estadísticamente significativas $(p<0.05)$ de sus medias entre los tipos de deshidratado en grupos para lugar de procedencia y tipo de deshidratado de pulpa de café. Cuando se empleó metanol $50 \%$ no presenta diferencias estadísticamente significativas para el tipo de deshidratado y cuando el lugar de procedencia fue Rodríguez de Mendoza y Utcubamba. Comparando los resultados de actividad antioxidante de las muestras de diferentes procedencias observamos en la Figura 5 que la actividad antioxidante fue mayor para la muestra de la provincia Utcubamba con $91.0 \%$, al respecto los polifenoles son componentes que aportan alto porcentaje de la capacidad antioxidante Vega et al. (2017). Se dice que la cantidad de fenoles es directamente proporcional a la capacidad antioxidante como es en el caso de la provincia de Utcubamba que presentó alto contenido en fenoles totales y capacidad antioxidante. Los resultados son cercanos a los de Valdez et al. (2018) que reportaron capacidad antioxidante de la pulpa de café de $93.89 \pm 3.85 \%$ de inhibición del radical DPPH. Para este estudio la AA es de mayor relevancia que los FT, debido a que en la AA expresa que la pulpa de café contiene componentes FT y no fenoles que también tienen propiedades 
antioxidantes. Sepúlveda y Zapata (2019) mencionan que los compuestos fenólicos, exhiben una amplia gama de propiedades biológicas como los antioxidantes que pueden ser vulnerables a la luz, temperatura, oxígeno, tipo de solvente y otros; así mismo, la estabilidad de la actividad antioxidante en función del tiempo se basa en la presencia de los compuestos fenólicos.

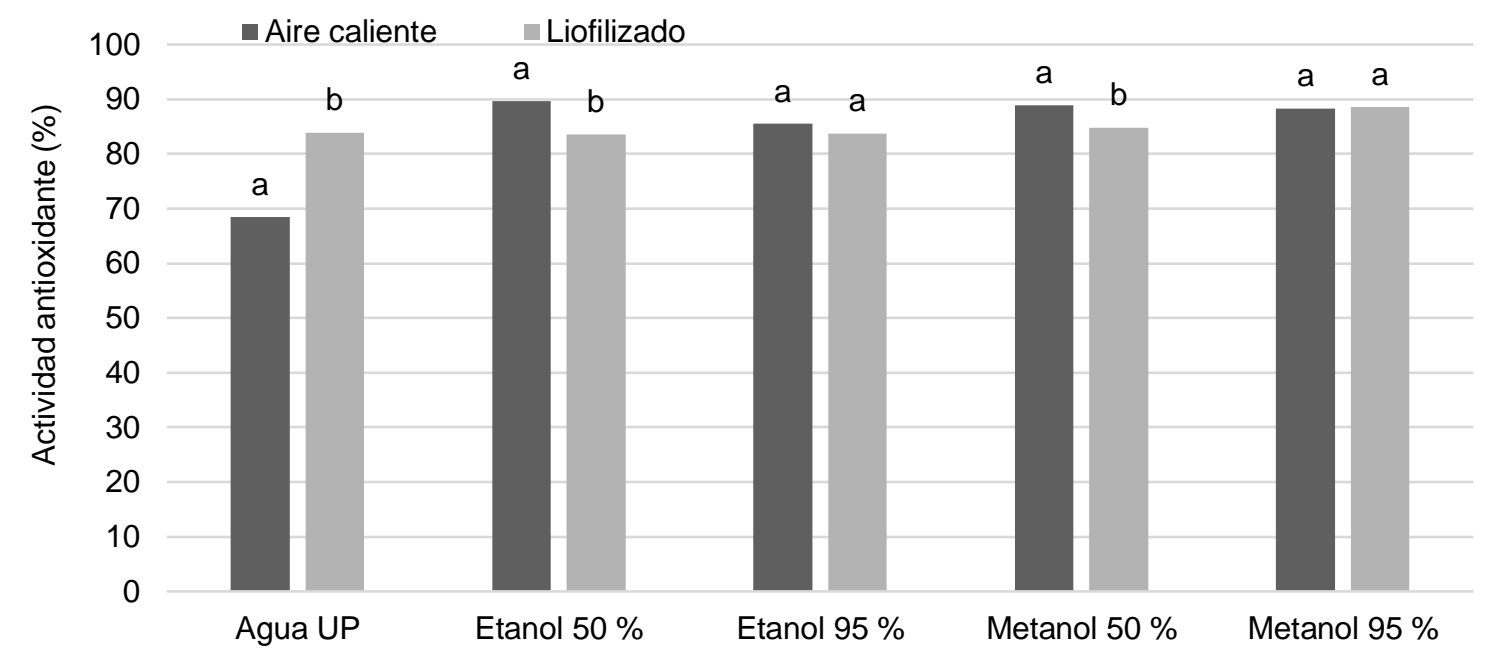

Tipos de solventes según concentraciones

Fig. 6: Actividad antioxidante de pulpa de café procedente de Luya. Para cada solvente, barras con letras diferentes indican diferencias significativas

El análisis de varianza y la prueba de Tukey mostraron que existe alta diferencia significativa a nivel de confianza del $95 \%$ para las procedencias de la pulpa de café; el análisis de varianza y la prueba Tukey mostraron que existe alta diferencia significativa a un nivel de confianza del $95 \%$ para los distintos solventes. Finalmente, se presentan en imágenes la pulpa de café molido según el tipo de deshidratación.

a.

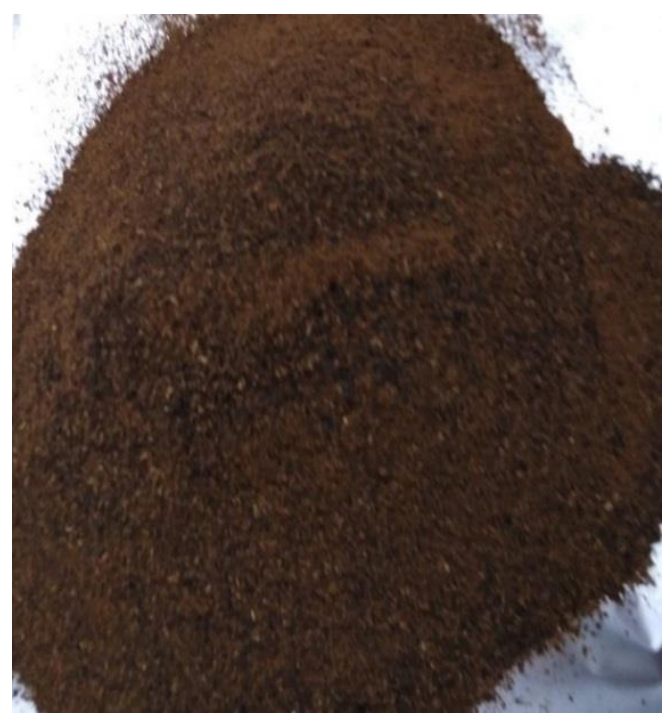

Fig. 7: Pulpa de café molido deshidratado.

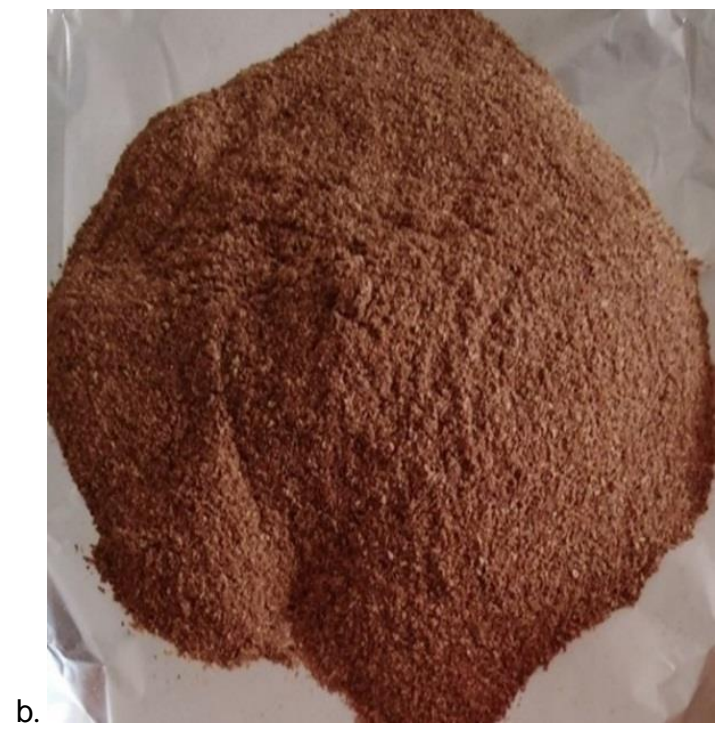

a) Secado por aire caliente; (b) Liofilización

\section{CONCLUSIONES}

Con base a los resultados de la presente investigación, para la pulpa de café variedad catimor deshidratada, se determinó una mayor capacidad de extracción cuando se emplea etanol $95 \%$ respecto a etanol $50 \%$ y metanol con purezas de $50 \%$ y $95 \%$; así como agua ultra pura. El contenido de fenoles totales es mayor cuando es deshidratado por liofilización que cuando es en aire caliente, además es en el café proveniente de la provincia de Utcubamba contuvieron más fenoles respecto a las provincias Luya y Rodríguez de Mendoza, y también las muestras de Utcubamba tuvo mayor capacidad antioxidante $(91 \%)$. Se demuestra que el café tiene compuestos bioactivos beneficiosos y saludables para las personas consumidoras. 


\section{NOTACIÓN}

DPPH: 2,2-difenil-1-picrilhidrazilo

AG: ácido gálico

PD: pulpa deshidratada

UP: ultra pura

\section{AGRADECIMIENTOS}

Este trabajo fue financiado por el CONCYTEC-FONDECYT en el marco de la convocatoria E041-01, CONTRATO 158-2018-FONDECYT-BM-IADT-SE y al Instituto de Investigación, Innovación y Desarrollo para el Sector Agrario y Agroindustrial de la Región Amazonas - IIDAA.

\section{REFERENCIAS}

Aboshora, W., Zhang, L., y otros seis autores, Effect of extraction method and solvent power on polyphenol and flavonoid levels in Hyphaene Thebaica L Mart (Arecaceae)(Doum) fruit, and its antioxidant and antibacterial activities, http://dx.doi.org/10.4314/tjpr.v13i12.16, Tropical Journal of Pharmaceutical Research, 13(12), 2057-2063 (2014)

Alkaltham, M., Ahmad, S., y Khizar, H., Determination of coffee fruit antioxidants cultivated in Saudi Arabia under different drying conditions, https://doi.org/10.1007/s11694-020-00378-4, Journal of Food Measurement and Characterization, 14(3), 1306-1313 (2020)

Chirinos, R., Rogez, H., y otros tres autores, Optimization of extraction conditions of antioxidant phenolic compounds from mashua (Tropaeolum tuberosum Ruíz \& Pavón) tubers, https://doi.org/10.1016/j.seppur.2006.12.005, Separation and Purification Technology, 55(2), 217-225 (2007)

Díaz, O., Ormaza, A., y Rojano, B., Effect of Coffee Roasting (Coffea arabica L. var. Castillo) on cup profile, Antioxidant Compound Content and Antioxidant Activity, https://dx.doi.org/10.4067/S0718-07642018000400031, Inf. Tecnol., 29(4), 3142 (2018)

Duangjai, A., Nungruthai, S., y otros cuatro autores, Comparison of antioxidant, antimicrobial activities and chemical profiles of three coffee (Coffea arabica L.) pulp aqueous extracts, https://doi.org/10.1016/j.imr.2016.09.001, Integrative Medicine Research, 5(4), 324-331 (2016)

Esquivel, P., y Jiménez, V.M., Functional properties of coffee and coffee by-products, https://doi.org/10.1016/j.foodres.2011.05.028, Food Research International, 46(2), 488-495 (2012)

Esquivel, P., Viñas, M., y otros seis autores, Coffee (Coffea arabica L.) by-Products as a Source of Carotenoids and Phenolic Compounds-Evaluation of varieties with different Peel Color, https://doi.org/10.3389/fsufs.2020.590597, Frontiers in Sustainable Food Systems, 4, 590597 (2020)

Fonseca-García, L., Calderón-Jaimes, L., y Rivera, M., Capacidad antioxidante y contenido de fenoles totales en café y subproductos del café producido y comercializado en norte de Santander (Colombia), Vitae, 21(3), 228-236 (2014)

Gemechu, F., Embracing nutritional qualities, biological activities and technological properties of coffee byproducts in functional food formulation, https://doi.org/10.1016/j.tifs.2020.08.005, Trends in Food Science \& Technology, 104, 235-261 (2020)

Gouri D, Sonam A., y otros cinco autores, Effect of extraction solvents on phenolic contents and antioxidant capacities of Artocarpus chaplasha and Carissa carandas fruits from Bangladesh, doi: 10.7324/JABB.2017.50307, Journal of Applied Biology \& Biotechnology, 5 (03), 39-044 (2017)

Heeger, A., Kosińska-Cagnazzo, A., Cantergiani, E., y Andlauer, W., Bioactives of coffee cherry pulp and its utilisation for production of Cascara beverage, https://doi.org/10.1016/j.foodchem.2016.11.067, Food chemistry, 221, 969-975 (2017)

Ji-Hee, K., Dong A., Jong, E., y Sun, M., Antioxidant effect of extracts from the coffee residue in raw and cooked meat, https://doi.org/10.3390/antiox5030021, Antioxidants, 5(3), 21 (2016)

Kamiyama, M., Joon-Kwan, M., Hae, J., y Takayuki, S., Role of degradation products of chlorogenic acid in the antioxidant activity of roasted coffee, https://doi.org/10.1021/jf5060563, Journal of agricultural and food chemistry, 63(7), 1996-2005 (2015)

Kim, J. H., Dong, A., Joon, E., y Soon, M., Antioxidant effect of extracts from the coffee residue in raw and cooked meat, https://doi.org/10.3390/antiox5030021, Antioxidants, 5(3), 21 (2016)

Kuskoski, E., García, A., y otros tres autores, Aplicação de diversos métodos químicos para determinar atividade antioxidante em polpa de frutas, https://doi.org/10.1590/S0101-20612005000400016, Food Science and Technology, 25(4), 726-732 (2005)

Park, J., Aislamiento y cuantificación de los principales ácidos clorogénicos en tres importantes marcas de café instantáneo y sus efectos potenciales sobre la despolarización y apoptosis de la membrana mitocondrial inducida por $\mathrm{H}_{2} \mathrm{O}_{2}$ en las células PC-12, https://doi.org/10.1039/c3fo60138b, Comida y función, 4 (11), 1632-1638 (2013) 
Peña-Aguilar, J., Murúa-Pagola, B., y otros cinco autores, Extracción y purificación de cafeína y ácido clorogénico de pulpa de beneficio húmedo de café, Investigación y Desarrollo en Ciencia y Tecnología de Alimentos, 2(1), 563-569 (2017)

Moreira, M., Melo, M.M., y otros cuatro autores, Solid coffee waste as alternative to produce carotenoids with antioxidant and antimicrobial activities, https://doi.org/10.1016/j.wasman.2018.10.017, Waste management, 82, 93-99 (2018)

Reichembach, L. H., y de Oliveira Petkowicz, C. L., Extraction and characterization of a pectin from coffee (Coffea arabica L.) pulp with gelling properties, https://doi.org/10.1016/j.carbpol.2020.116473, Carbohydrate Polymers, 245, 116473 (2020)

Serna-Cock, L., Cristian Torres-León, C. y Ayala-Aponte, A., Efecto de la adición de Edulcorantes no calóricos sobre las Propiedades Fisicoquímicas y la Cinética de Secado de cáscara de Mango Liofilizado, doi:10.4067/S071807642015000400006, Inf. Tecnol., 26(4), 37-44 (2015)

Serna-Jiménez, J. A., Torres-Valenzuela, L. S., Martínez-Cortínez, K., y Hernández-Sandoval, M. C., Uso de pulpa de café como alternativa para la valorización de subproductos, http://dx.doi.org/10.18273/revion.v31n1-2018006, Revista ION, 31(1), 37-42 (2018)

Sepúlveda, C., y Zapata, J., Efecto de la Temperatura, el pH y el contenido en Sólidos sobre los Compuestos Fenólicos y la Actividad Antioxidante del Extracto de Bixa orellana L., http://dx.doi.org/10.4067/S0718-07642019000500057. Inf. Tecnol., 30(5), 57-66 (2019)

Sushant, A., Manu, K., Krisha, D., y otros tres autores, Total Phenolic Content, Flavonoid Content and Antioxidant Potential of Wild Vegetables from Western Nepal, https://doi.org/10.3390/plants8040096, Plants, 8, 96 (2019)

Tran, T. M. K., Kirkman, T., Nguyen, M., y Van Vuong, Q., Effects of drying on physical properties, phenolic compounds and antioxidant capacity of Robusta wet coffee pulp (Coffea canephora), https://doi.org/10.1016/j.heliyon.2020.e04498, Heliyon, 6(7), e04498 (2020)

Singleton, V. L., Orthofer, R., y Lamuela-Raventós, R. M., Analysis of total phenols and other oxidation substrates and antioxidants by means of folin-ciocalteu reagent, https://doi.org/10.1016/S0076-6879(99)99017-1, Methods in enzymology, 299, 152-178 (1999)

Soquetta, M., Terra, L., y Bastos, C., Green technologies for the extraction of bioactive compounds in fruits and vegetables, https://doi.org/10.1080/19476337.2017.1411978, CyTA-Journal of Food, 16(1), 400-412 (2018)

Valdez R. B., Oseguera S. E., y otros cuatro autores, Efecto del tiempo de almacenamiento en el contenido de compuestos bioactivos presentes en la pulpa de café orgánico. Investigación y Desarrollo en Ciencia y Tecnología de Alimentos, 3(1), 692-696 (2018)

Vega, Aracelly, De León, Javier A, y Reyes, Stephany M., Determinación del contenido de polifenoles totales, flavonoides y actividad antioxidante de 34 cafés comerciales de Panamá, https://dx.doi.org/10.4067/S0718-07642017000400005, Inf. Tecnol., 28(4), 29-38 (2017) 